CANCER THERAPEUTICS

\section{Fomenting radical behavior}

Nature $\mathbf{4 7 5}, 231-234$ (2011)

After malignant transformation, cells show molecular signatures-such as increased levels of reactive oxygen species (ROS) - indicative of stress pathways. To adapt to such persistent stressors, transformed cells rely upon stress response pathways much more than do normal cells. Raj et al. now show that targeting components of the ROS homeostasis network may offer an effective means for killing cancer cells. Using chemical screening, the authors identified the plant natural product piperlongumine as an inducer of cancer cell apoptosis that confers no toxicity to normal cells. Proteomic analysis and in vitro assays revealed that piperlongumine binds and inhibits the activity of glutathione S-transferase pi (GSTPI), a component of a protein complex involved in ROS homeostasis. Measurements of cellular ROS levels revealed that piperlongumine treatment enhances hydrogen peroxide and nitric oxide production in cells with a cancer genotype but not in normal cells; similarly, the compound's effects are abrogated when it is administered together with a reducing agent such as $\mathrm{N}$-acetyl-L-cysteine or when GSTPI is overexpressed. These results support a model in which piperlongumine-induced perturbation of ROS levels leads to selective apoptosis of transformed cells that have become dependent on the ROS stress response. The authors also showed in tumor xenografts and mouse cancer models that piperlongumine has promise for therapeutic applications.

\section{ENZYME MECHANISMS}

\section{A cope-ing strategy}

J. Am. Chem. Soc., published online 21 July 2011 doi:10.1021/ja2034969

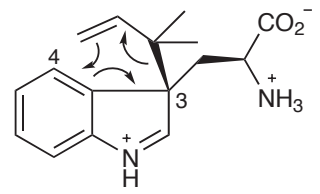

Dimethylallyltryptophan (DMAT), generated by DMAT synthase, is an alkaloid with a prenyl group attached to the C-4 position of a tryptophan indole ring. The attachment of the prenyl group to the less reactive $\mathrm{C}-4$ indole position instead of the more nucleophilic C-3 position has been suggested to occur through either of two mechanisms, one involving a Cope rearrangement to transfer the alkyl group from C-3 to C-4 during the reaction. However, direct evidence for this proposal was lacking. Luk et al. now identify a relevant intermediate in their analysis of an active site mutant of DMAT synthase. Lys174 had previously been suggested to catalyze the final step of the second proposed mechanism, in which the prenyl group is connected directly to the C-4 position from the beginning. Kinetic analysis of a Lys174Ala mutant did show a decrease in $k_{\text {cat }}$ consistent with this role. However, characterization of the reaction products surprisingly indicated the prenyl group was attached at C-3, and its connectivity was perfectly poised for a Cope rearrangement to yield the normal product. Finally, analysis of an existing crystal structure of the enzyme in complex with a nonhydrolyzable analog provided no rationale for how the enzyme might drive reactivity at the unfavored C-4 investigation into this interesting enzyme. $C G$

SIGNALING

\section{Hijacking a GTPase \\ Science 333, 453-456 (2011)}

The intracellular pathogen Legionella pneumophila exploits host-cell trafficking machinery during infection by modulating the activity of the GTPase Rab1; the bacterial protein SidM recruits Rab1 to L. pneumophila-containing vacuoles (LCVs) and adds AMP to Tyr77, transiently locking Rab1 in an active, GTP-bound state. Release of Rab1 from LCVs, however, requires its inactivation. Now Neunuebel et al. have identified SidD, a bacterially encoded enzyme that de-AMPylates Rab1. The authors noted a conserved open reading frame adjacent to sid $M$ called sidD; deletion of sidD eliminated Rab1 de-AMPylase activity from L. pneumophila lysate. Recombinant position. Together, these data call for further

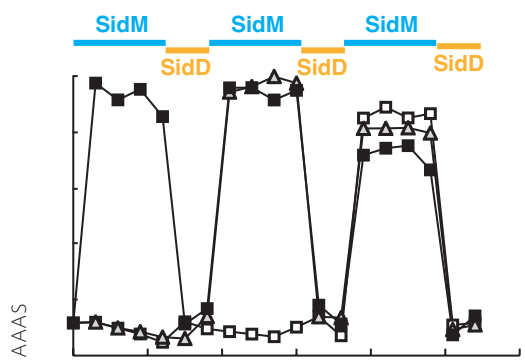

SidD catalyzed removal of $\left[{ }^{32} \mathrm{P}\right]$ AMP from AMPylated Rab1 in vitro in a concentrationdependent manner and, together with SidM, catalyzed an AMPylation-deAMPylation cycle for Rab1, the products of which were validated by MS. In cells, expression of SidD counteracted Golgi fragmentation phenotypes associated with ectopic SidM expression and restored interaction between GTP-Rab1 and regulatory GTPaseactivating proteins. During infection, $\Delta$ sidD LCVs showed prolonged colocalization with Rab1 compared to wild-type LCVs, suggesting delayed release of Rab1 from vesicles in the absence of SidD activity. Together, these data support a model in which L. pneumophila AMPylates-and thereby stably activates-host Rab1 to promote docking and fusion of secretory vesicles with LCVs until bacterially encoded SidD de-AMPylates Rab1, allowing Rab1 inactivation and membrane extraction. $A D$

\section{LIPIDOMICS}

\section{A viral egress}

Proc. Natl. Acad. Sci. USA, published online 18 July 2011, doi:10.1073/pnas.1109796108

Infection of human fibroblast cells with the enveloped virus human cytomegalovirus (HCMV) upregulates various components of central carbon metabolism, including fatty acids. Using a lipidomics approach, Liu et al. now show that, in addition to specific glycerophospholipid changes, virion membranes acquire a unique profile that hints at their origin beyond their assembly in a virus-induced organelle. The authors monitored 146 unique glycerophospholipids, including phosphatidic acid (PA) species, phosphatidylethanolamines (PEs) and plasmalogens, over the course of the HMCV replication cycle. They found that the virion glycerophospholipid composition induced by the virus is distinct from that of infected cells and other viral lipidomes that have been studied, including that of HIV-1. However, virion membranes in HCMV-infected cells did resemble those of synaptic vesicles. RNAi knockdown of a critical exocytosis mediator in fibroblasts, SNAP-23, resulted in a severe decrease in $\mathrm{HMCV}$ production. These data suggest that HMCV induces and buds into exosome-like vesicles, which are distinct from any existing cellular membrane and which fuse to the plasma membrane of the host cells to release the virions to the extracellular space. $M B$

Written by Mirella Bucci, Amy Donner, Catherine Goodman \& Terry L. Sheppard 